\title{
Prediction of 3D ground reaction forces during gait based on accelerometer data
}

\author{
Gustavo Leporace ${ }^{1,2 *}$, Luiz Alberto Batista ${ }^{3}$, Jurandir Nadal ${ }^{1}$ \\ ${ }^{1}$ Biomedical Engineering Program, Federal University of Rio de Janeiro, Rio de Janeiro, RJ, Brazil. \\ ${ }^{2}$ Institute Brasil of Health Technologies, Rio de Janeiro, RJ, Brazil. \\ ${ }^{3}$ Laboratory of Biomechanics and Motor Behavior, Federal University of Rio de Janeiro, Rio de Janeiro, RJ, Brazil.
}

\begin{abstract}
Introduction: The aim of this study was to predict 3D ground reaction force signals based on accelerometer data during gait, using a feed-forward neural network (MLP). Methods: Seventeen healthy subjects were instructed to walk at a self-selected speed with a $3 \mathrm{D}$ accelerometer attached to the distal and anterior part of the shank. A force plate was embedded into the middle of the walkway. MLP neural networks with one hidden layer and three output layers were selected to simulate the anteroposterior (AP), vertical (Vert) and mediolateral (ML) ground reaction forces (GRF). The input layer was composed of fourteen inputs obtained from accelerometer signals, selected based on previous studies. Principal component analysis (PCA) was used to compare the simulated and collected curves. The Pearson correlation coefficient and the mean absolute deviation (MAD) between signals were calculated. Results: PCA identified small, but significant differences between collected and simulated signals in the loading response phases of AP and ML GRF, while Vert did not show differences. The correlation between the simulated and collected signals was high (AP: 0.97; Vert: 0.98; ML: 0.80). MAD was $1.8 \% \mathrm{BW}$ for AP, $4.5 \% \mathrm{BW}$ for Vert and $1.4 \% \mathrm{BW}$ for ML. Conclusion: This study confirmed that multilayer perceptron neural network can predict the highly non-linear relationship of shank acceleration parameters and ground reaction forces, as well as other studies have done using plantar pressure devices. The greater advantages of this device are the low cost and the possibility of use outside the laboratory environment.
\end{abstract}

Keywords Acceleration, Force, Gait, Feedforward neural network, Simulation.

\section{Introduction}

Gait analysis has been proposed as an alternative for evaluating the functionality of lower limbs during daily living tasks (Baker, 2006). The basic instrumentation usually recommended is a motion analysis system (MAS), force plates and electromyography. All these instruments have high costs, which makes it difficult to implement a gait service in the clinical practice context, outside the academic laboratory. Therefore, alternatives for low cost equipment have been proposed. Inertial sensors have been used to replace the MAS (Favre et al., 2009) and seem to be a promising technology. Several studies have already shown the clinical applicability of these

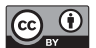

This is an Open Access article distributed under the terms of the Creative Commons Attribution License, which permits unrestricted use, distribution, and reproduction in any medium, provided the original work is properly cited.

How to cite this article: Leporace G, Batista LA, Nadal J. Prediction of 3D ground reaction forces during gait based on accelerometer data. Res Biomed Eng. 2018; 34(3):211-216. DOI: 10.1590/2446-4740.06817

*Corresponding author: Rua Visconde de Pirajá, 595, room 1201, Ipanema, CEP 22410-003, Rio de Janeiro, RJ, Brazil. E-mail: gustavo@biocinetica.com

Received: 01 November 2017 / Accepted: 05 July 2018 systems to collect kinematic data (Fasel et al., 2015; Peruzzi et al., 2011).

However, little effort has been made to replace force plates to acquire ground reaction force (GRF). So far, there is no means of directly measuring these forces during walking. Computational mathematical systems have been developed to estimate these forces by optimization algorithms based on ground reaction forces and kinematic data (Lewis \& Garibay, 2015). Usually, data from optoelectronic motion analysis systems and force plates are used to calculate inverse and forward dynamics. However, these devices limit the performance of the gait analysis to a laboratory environment - besides its high cost (Baker, 2006) reducing the application of quantitative biomechanical tests in the clinical practice.

Some studies have proposed a model to predict GRF based on insole plantar pressure measurements (Fong et al., 2008; Jung et al., 2014), presenting promising results. Despite the advance, the required insoles still have an elevated cost and do not allow the analysis to be done barefoot, which can influence the outcome (Chen et al., 2015). Accelerometers attached to the tibia, on the other hand, are lightweight and have already been used to 
simulate some discrete force variables, using regression analysis (Elvin et al., 2007). The limitation of these latter models is that they do not estimate the complete GRF signal. To overcome these limitations, some studies have used artificial neural networks to predict moment of force data during different tasks (Favre et al., 2012; Oh et al., 2013).

Therefore, the aim of this study was to predict 3D ground reaction force signals based on accelerometer data during gait, using a feed-forward neural network (MLP). The hypothesis of this study was that $3 \mathrm{D}$ accelerometer data from the distal tibia would allow an adequate simulation of GRF, with prediction errors comparable to other studies using a plantar pressure device.

\section{Methods}

Seventeen healthy subjects ( 11 males; $27.1 \pm 3.4$ years old; $84.3 \pm 4.5 \mathrm{~kg}$ ) participated in the study. All of them provided written consent, approved along with the experimental protocol of the Institutional Ethics and Research Committee. Inclusion criteria were: (i) to have no history of ligament injuries, nor pain at the time of the tests; (ii) to be between 20 and 40 years old; and (iii) to walk independently, without the need of any orthoses or braces.

Subjects were instructed to walk at a self-selected speed along an eight $\mathrm{m}$ long walkway. Each subject performed six laps with a $3 \mathrm{D}$ accelerometer ( \pm 6 g, model MMA7260Q, Freescale, USA) attached to the distal and anterior part of the shank. A force plate (AccuGait, AMTI, USA) was embedded into the middle of the walkway. The first two laps were not collected to allow familiarization with the task. The last four laps were collected in order to capture, during four gait cycles the acceleration of the shank. All data were collected simultaneously using a BIOPAC system (UIM, MP100 Systems, BIOPAC, USA) with a sample rate of $1 \mathrm{kHz}$.

After collection, the data were filtered by a $2^{\text {nd }}$ order Butterworth low pass filter, applied in the direct and reverse directions to avoid phase shift. The cutoff frequency for GRF and accelerometer data was $25 \mathrm{~Hz}$. Stance phase was defined when vertical ground reaction forces were above $10 \mathrm{~N}$.

To fit anteroposterior (AP), vertical (Vert) and mediolateral (ML) GRF curves, fourteen inputs were selected based on previous studies (Favre et al., 2012; Liu et al., 2009), as follows:

$\mathrm{i}$ to iii: the 3D leg acceleration data normalized by body weight; iv to vi: the 3D leg velocity, represented by the single integration of the acceleration curve;

vii to ix: 3D leg displacement, represented by the double integration of the acceleration curve;

$\mathrm{x}$ to xii: the first derivate of the $3 \mathrm{D}$ accelerometer signal

xiii: the stance duration (s); and

xiv: time point of the stance phase of gait (\%), expressed as a percentage of the stance time.

Multilayer perceptron (MLP) neural networks with one hidden layer and three output layers were selected to simulate the 3D GRF. The number of neurons in the hidden layer was chosen by testing the fitting of signals with different sizes, always seeking the most parsimonious model, i.e. few hidden neurons and good generalization power. Networks were modeled using from five to 12 neurons in the hidden layer.

To assess the network fitting, a leave one out cross-validation strategy was used. The network was trained using the Levenberg-Marquardt backpropagation algorithm according to Favre et al. (2012) and Liu et al. (2009).

Hyperbolic tangent and linear activation transfer functions were used in the hidden and output layers, respectively. These typologies were used in previous biomechanical studies which showed that these transfer functions produced the best signal prediction (Favre et al., 2012; Liu et al., 2009). To avoid overfitting, the generalization error obtained for the validation set during the training process, and the minimum gradient were used as stop criteria.

The network with the lowest mean absolute deviation (MAD) among all subjects was selected as the best number of neurons in the hidden layers. MAD was calculated as:

$\mathrm{MAD}=\frac{1}{\mathrm{~N}} \sum_{\mathrm{t}=1}^{\mathrm{N}}|\widehat{\operatorname{GRF}}(\mathrm{t})-\operatorname{GRF}(\mathrm{t})|$

where $\widehat{\mathrm{GRF}}(\mathrm{t})$ represents the simulated ground reaction force, $\operatorname{GRF}(\mathrm{t})$ corresponds to the collected GRF and $\mathrm{N}$ is the vector size.

The normalized mean absolute deviation (MAD\%) was also calculated as [8]:

$$
\mathrm{MAD} \%=\frac{\mathrm{MAD}}{\operatorname{range}(\operatorname{GRF}(\mathrm{t}))} * 100
$$

Data from each gait cycle were interpolated into 51 values, representing $0 \%$ to $100 \%$ of the stance phase. To test significant differences between the simulated and measured signals, the $136 \mathrm{GRF}$ curves, 68 measured and 68 simulated, were inserted into a matrix D [136 x 51], 
where each row corresponded to a gait cycle and each column corresponded to the interpolated signals.

Principal Component (PC) analysis was applied to three independent matrices $\mathbf{D}$, each one corresponding to a different GRF component (Jolliffe, 2002). Initially, the mean was subtracted, the covariance matrix $\mathbf{S}$ [51 x 51] was calculated and, finally, the eigenvectors and eigenvalues were estimated from $\mathbf{S}$, based on a singular value decomposition algorithm (Jolliffe, 2002). The number of PC retained in the analysis represented approximately $80 \%$ of the original data variance. The scores of the retained PCs were compared between the simulated and measured signals using a paired t-test. The correspondent eigenvectors of the statistically significant PC scores were analyzed in temporal correspondence to the original signals to identify the location where the variance between them could be explained (Leporace et al., 2012). The locations where eigenvectors deviate from zero indicate increased differences between the simulated and collected signals.

The correlation between the simulated and collected signal was also calculated for each GRS component, using the Pearson's correlation coefficient. The significance level was set at 0.05 . The effect size was also calculated based on Cohen (1988). Values higher than 0.8 were considered large and values lower than 0.5 were considered small.

\section{Results}

The number of neurons in the hidden layer with the lowest MAD was ten. The vertical ground reaction forces showed the lowest errors, followed by the anteroposterior and, with the greatest errors, the mediolateral forces. The correlation between the simulated and collected signals was high, with values above 0.8 (Table 1).

The simulation of the signal showed good qualitative results, with all the simulated signals resembling the original data (Figures 1 to 3 ). Four, five and four PCs were retained for the vertical, anteroposterior and mediolateral GRF comparison between the signals, respectively. The vertical GRF showed no significant differences $(\mathrm{p}>0.05)$, with low effect size (Table 2, Figure 2). The anteroposterior GRF showed significant differences in the fifth PC $(p=0.009)$ (Table 2, Figure 3), and the mediolateral forces presented differences in the second $(\mathrm{p}=0.01)$ and third $(\mathrm{p}=0.02)$ PCs (Table 2, Figure 3). The loading factors indicated that the variance was higher in the first $15 \%$ of the stance phase, representing the impact phase and the first active peak.
Table 1. Mean absolute deviation (MAD) and normalized mean absolute deviation (\%MAD), expressed as mean ( \pm standard deviation), and Pearson Correlation Coefficient (r).

\begin{tabular}{lccc}
\hline & MAD $( \pm$ sd) & \% MAD ( \pm sd) & r \\
\hline Anteroposterior & $1.8 \pm 0.3 \% \mathrm{BW}$ & $4.6 \pm 0.7 \%$ & 0.97 \\
Vertical & $4.5 \pm 1.1 \% \mathrm{BW}$ & $4.0 \pm 0.8 \%$ & 0.98 \\
Mediolateral & $1.4 \pm 0.3 \% \mathrm{BW}$ & $10.5 \pm 3.3 \%$ & 0.80 \\
\hline
\end{tabular}

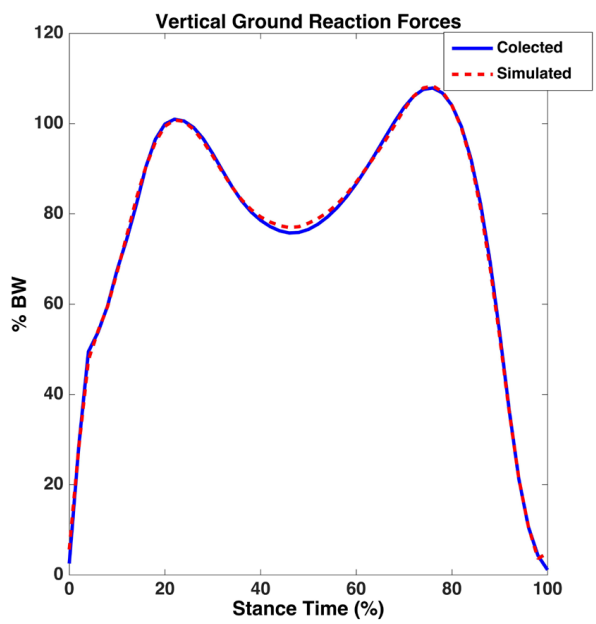

Figure 1. Vertical Ground Reaction Forces. The black continuous line represents the collected signal and the magenta dashed line represents the simulated signal. The area surrounding the averages represents $95 \% \mathrm{CI}$ for each group.
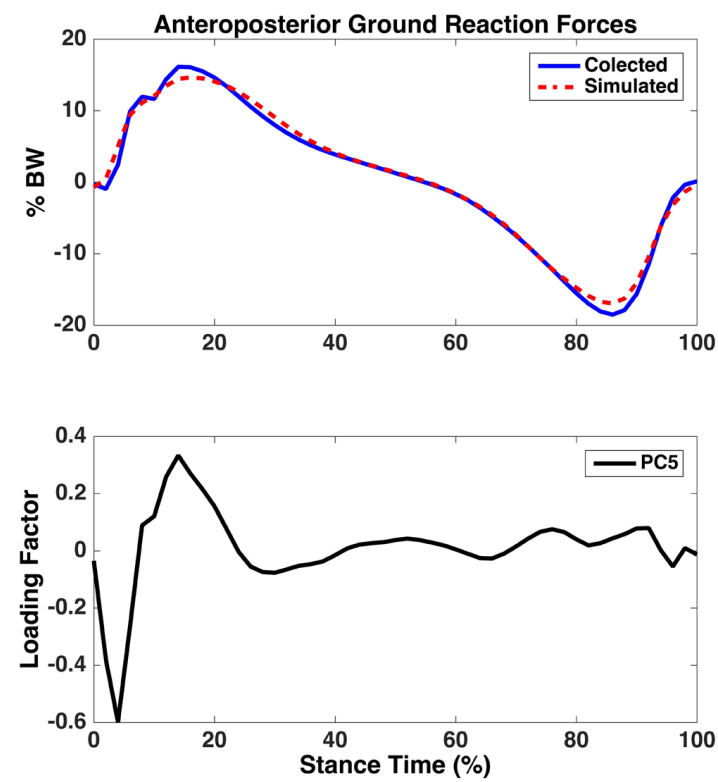

Figure 2. Anteroposterior Ground Reaction Forces. Above: The black continuous line represents the collected signal and the magenta dashed line represents the simulated signal. The area surrounding the averages represents $95 \% \mathrm{CI}$ for each group. Below: Fifth principal component, and the arrows represent the locations where there is higher variance, representing differences between signals. 
Table 2. P value and effect size (ES) of the comparison between the collected and simulated ground reaction forces.

\begin{tabular}{|c|c|c|c|c|c|c|}
\hline & \multicolumn{2}{|c|}{ Anteroposterior } & \multicolumn{2}{|c|}{ Vertical } & \multicolumn{2}{|c|}{ Mediolateral } \\
\hline & p value & ES & p value & ES & p value & ES \\
\hline PC1 & 0.07 & 0.44 & 0.48 & 0.12 & 0.31 & 0.30 \\
\hline PC2 & 0.95 & 0.01 & 0.93 & 0.02 & $0.01 * *$ & 1.04 \\
\hline PC3 & 0.08 & 0.63 & 0.71 & 0.09 & $0.02 *$ & 1.06 \\
\hline PC4 & 0.50 & 0.20 & 0.98 & 0.01 & 0.24 & 0.46 \\
\hline PC5 & $0.009 * *$ & 0.94 & - & - & - & - \\
\hline
\end{tabular}

$* \mathrm{p} \leq 0.05 ; * * \mathrm{p} \leq 0.01 . \mathrm{PC}=$ Principal Component; $\mathrm{ES}=$ Effect Size.
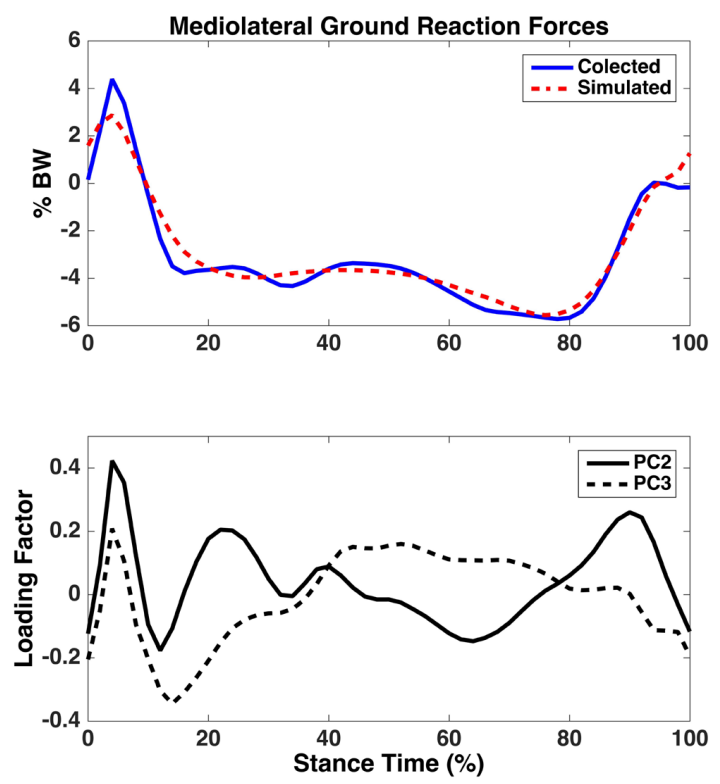

Figure 3. Mediolateral Ground Reaction Forces. Above: The black continuous line represents the collected signal and the magenta dashed line represents the simulated signal. The area surrounding the averages represents $95 \% \mathrm{CI}$ for each group. Below: Second (continuous line) and third (dashed line) principal components, and the arrows represent the locations where there is higher variance, representing differences between signals.

\section{Discussion}

The purpose of this study was to predict 3D ground reaction forces during gait based on a lightweight and low cost accelerometer attached to the distal tibia. The results showed that the neural network model achieved success to simulate the shape of the 3D GRF curves, although the PC analysis still discriminated some differences between the simulated and collected signal.

The MAD and MAD\% were equal or lower than other studies that used plantar pressure insoles to calculate GRF. The MAD from AP GRF simulation from studies using plantar pressure insoles ranged from 1.6 to $5.8 \% \mathrm{BW}$, while the $\% \mathrm{MAD}$ ranged from $7.3 \%$ to $10.1 \%$ and the correlation coefficient ranged from 0.85 to 0.98 (Fong et al., 2008; Forner-Cordero et al., 2004; Jung et al., 2014; Liedtke et al., 2007; Rouhani et al., 2010; Savelberg \& Lange, 1999). The MAD, MAD\% and correlation coefficient found in the present study were $1.8 \% \mathrm{BW}, 4.6 \%$ and 0.97 , respectively. Vert GRF simulation in the literature has error values ranging from $6.6 \% \mathrm{BW}$ to $13.8 \% \mathrm{BW}$; the relative error ranging from $7.3 \%$ to $10.1 \%$, while the present study showed values of $4.5 \% \mathrm{BW}$ and $4.0 \%$. The correlation coefficient of all studies is close, ranging from 0.97 to 0.99 , similar to the neural network simulation (Barnett et al., 2001; Fong et al., 2008; Forner-Cordero et al., 2004; Jung et al., 2014; Liedtke et al., 2007; Oh et al., 2013; Rouhani et al., 2010).

Similar to other studies, ML GRF presented lower correlation and higher error than AP and Vert GRF (Liedtke et al., 2007; Oh et al., 2013). The ML error of this study was similar to Oh et al. (2013), showing a relative error of $10 \%$. This should be due to the greater variability of this signal (John et al., 2012). Maybe, with a higher sample size this variability could be captured by the neural network model, decreasing the error.

Despite the similarity of the error, none of the studies have validated their models, making it difficult to discuss the fitness of the model, not allowing to confirm if the model is well fitted or that it cannot be improved without a validation process. In a previous study (Leporace et al., 2015), it has been shown, through a residual analysis, the closeness of the model proposed in the present study to a normal probability distribution, confirming the data is suitable.

Additionally, this is the first study to compare the simulated and collected GRF using a statistical technique that is more sensitive to differences than more traditional ones (Leporace et al., 2012; Muniz et al., 2010). The advantage of using PCA is that it allows the analysis of the entire time series, not just discrete values extracted from them (Jolliffe, 2002).

The simulated and collected vertical ground reaction forces (Figure 2) showed no differences, suggesting that the use of this curve in clinical practice is acceptable. 
This result is very important, since some variables extracted from vertical GRF have already been linked with injury risk (Zadpoor \& Nikooyan, 2011) and disease progression (Houck et al., 2011); and this system is low cost and does not need a highly instrumented biomechanics laboratory to collect data. On the other hand, PCA showed that AP and ML curves still present differences that could influence the use of these signals in clinical practice. The loading factor from the AP and ML GRF found differences in $5 \%$ and $15 \%$ of the stance phase, representing a decreased impact peak and first active peak.

The impact forces' peak had a higher frequency range (10 to $20 \mathrm{HZ}$ ) and represents the rapid deceleration of the leg and foot just after the initial contact with the ground, while the active peak had a lower frequency range $(4$ to $8 \mathrm{~Hz}$ ), representing voluntary lower extremity motion and the deceleration of the center of mass $(\mathrm{CoM})$ (Gruber et al., 2014). It is possible that the deceleration of the CoM and foot could not be captured, since in the present study only one accelerometer was attached to the shank. It was proposed, for future studies, the inclusion of sensors on the sacrum, representing the acceleration of the CoM, and on the foot, providing more information for the neural networks to simulate the GRF signals.

This study confirmed that multilayer perceptron neural network can predict the highly non-linear relationship of shank acceleration parameters and ground reaction forces in healthy young individuals, as well as other studies have done using plantar pressure devices. The greater advantages of this device are the low cost and the possibility of use outside the laboratory environment. Although the error rates were low, ranging from 4 to $10 \%$, some improvements must be made, regarding the management of input parameters and number of sensors used, prior to application in clinical settings.

The results of this study must be only applied to level walking of healthy individuals. Although it is known that straight-line level walking is actually not common in real-world activities (Orendurff et al., 2008), usually level gait with self-selected speed is the most used task in clinical biomechanical gait assessments aimed at identifying lower limb dysfunctions. Future studies must be performed applying this algorithm to different orthopedic and neurologic conditions.

\section{Acknowledgements}

The authors would like to thank Felipe Alvim, Aluizio Netto, and Igor Jesus for the help in data collection.

This study was partially supported by the Brazilian Research Council (CNPq), Carlos Chagas Filho Foundation for Research Support of Rio de Janeiro (FAPERJ) and Coordenação de Aperfeiçoamento de Pessoal de Nível Superior (CAPES).

\section{References}

Baker R. Gait analysis methods in rehabilitation. J Neurol Rehabil. 2006; 2:3-4. PMid:16512912.

Barnett S, Cunningham JL, West S. A comparison of vertical force and temporal parameters produced by an in-shoe pressure measuring system and a force platform. Clin Biomech (Bristol, Avon). 2001; 16(4):353-7. http://dx.doi.org/10.1016/S02680033(01)00026-2. PMid:11358623.

Chen JP, Chung MJ, Wu CY, Cheng KW, Wang MJ. Comparison of barefoot walking and shod walking between children with and without flat feet. J Am Podiatr Med Assoc. 2015; 105(3):218-25. http://dx.doi.org/10.7547/0003-0538-105.3.218. PMid:26146967.

Cohen J. Statistical power analysis for the behavioral sciences 2nd ed. New York: Lawrence Erlbaum Associates; 1988.

Elvin NG, Elvin AA, Arnoczky SP. Correlation between ground reaction force and tibial acceleration in vertical jumping. $\mathrm{J}$ Appl Biomech. 2007; 23(3):180-9. http://dx.doi.org/10.1123/ jab.23.3.180. PMid:18089915.

Fasel B, Favre J, Chardonnens J, Gremion G, Aminian K. An inertial sensor-based system for spatio-temporal analysis in classic cross-country skiing diagonal technique. J Biomech. 2015; 48(12):3199-205. http://dx.doi.org/10.1016/j. jbiomech.2015.07.001. PMid:26209087.

Favre J, Aissaoui R, Jolles BM, de Guise JA, Aminian K. Functional calibration procedure for 3D knee joint angle description using inertial sensors. J Biomech. 2009; 42(14):2330-5. http:// dx.doi.org/10.1016/j.jbiomech.2009.06.025. PMid:19665712.

Favre J, Hayoz M, Erhart-Hledik JC, Andriacchi TP. A neural network model to predict knee adduction moment during walking based on ground reaction force and anthropometric measurements. J Biomech. 2012; 45(4):692-8. http://dx.doi. org/10.1016/j.jbiomech.2011.11.057. PMid:22257888.

Fong DT, Chan YY, Hong Y, Yung PS, Fung KY, Chan KM. Estimating the complete ground reaction forces with pressure insoles in walking. J Biomech. 2008; 41(11):2597-601. http:// dx.doi.org/10.1016/j.jbiomech.2008.05.007. PMid:18571656.

Forner-Cordero A, Koopman HJ, van der Helm FC. Use of pressure insoles to calculate the complete ground reaction forces. J Biomech. 2004; 37(9):1427-32. http://dx.doi.org/10.1016/j. jbiomech.2003.12.016. PMid:15275851.

Gruber AH, Boyer KA, Derrick TR, Hamill J. Impact shock frequency components and attenuation in rearfoot and forefoot running. J Sport Health Sci. 2014; 3(2):113-21. http://dx.doi. org/10.1016/j.jshs.2014.03.004.

Houck J, Kneiss J, Bukata SV, Puzas JE. Analysis of vertical ground reaction force variables during a Sit to Stand task in participants recovering from a hip fracture. Clin Biomech (Bristol, Avon). 2011; 26(5):470-6. http://dx.doi.org/10.1016/j. clinbiomech.2010.12.004. PMid:21196069.

John CT, Seth A, Schwartz MH, Delp SL. Contributions of muscles to mediolateral ground reaction force over a range of walking speeds. J Biomech. 2012; 45(14):2438-43. http:// dx.doi.org/10.1016/j.jbiomech.2012.06.037. PMid:22884038. 
Jolliffe IT. Principal component analysis. New York: SpringerVerlag; 2002.

Jung Y, Jung M, Lee K, Koo S. Ground reaction force estimation using an insole-type pressure mat and joint kinematics during walking. J Biomech. 2014; 47(11):2693-9. http://dx.doi. org/10.1016/j.jbiomech.2014.05.007. PMid:24917473.

Leporace G, Batista LA, Metsavaht L, Nadal J. Residual analysis of ground reaction forces simulation during gait using neural networks with different configurations. In: Conference Proceedings of the IEEE Engineering and Medical Biology Society; Aug 25-29; Milan, Italy. USA: IEEE; 2015. p. 281215. http://dx.doi.org/10.1109/EMBC.2015.7318976.

Leporace G, Batista LA, Muniz AM, Zeitoune G, Luciano T, Metsavaht L, Nadal J. Classification of gait kinematics of anterior cruciate ligament reconstructed subjects using principal component analysis and regressions modelling. In: Conference Proceedings of the IEEE Engineering and Medical Biology Society; 2012 Aug 28-Sept 1; San Diego, CA. USA: IEEE; 2012. p. 6514-7. http://dx.doi.org/10.1109/ EMBC.2012.6347486.

Lewis CL, Garibay EJ. Effect of increased pushoff during gait on hip joint forces. J Biomech. 2015; 48(1):181-5. http:// dx.doi.org/10.1016/j.jbiomech.2014.10.033. PMid:25468661.

Liedtke C, Fokkenrood SA, Menger JT, van der Kooij H, Veltink PH. Evaluation of instrumented shoes for ambulatory assessment of ground reaction forces. Gait Posture. 2007; 26(1):39-47. http://dx.doi.org/10.1016/j.gaitpost.2006.07.017. PMid:17010612.

Liu Y, Shih SM, Tian SL, Zhong YJ, Li L. Lower extremity joint torque predicted by using artificial neural network during vertical jump. J Biomech. 2009; 42(7):906-11. http://dx.doi. org/10.1016/j.jbiomech.2009.01.033. PMid:19261287.
Muniz AM, Liu H, Lyons KE, Pahwa R, Liu W, Nobre FF, Nadal J. Comparison among probabilistic neural network, support vector machine and logistic regression for evaluating the effect of subthalamic stimulation in Parkinson disease on ground reaction force during gait. J Biomech. 2010; 43(4):720-6. http:// dx.doi.org/10.1016/j.jbiomech.2009.10.018. PMid:19914622.

Oh SE, Choi A, Mun JH. Prediction of ground reaction forces during gait based on kinematics and a neural network model. J Biomech. 2013; 46(14):2372-80. http://dx.doi.org/10.1016/j. jbiomech.2013.07.036. PMid:23962528.

Orendurff MS, Schoen JA, Bernatz GC, Segal AD, Klute GK. How humans walk: bout duration, steps per bout, and rest duration. J Rehabil Res Dev. 2008; 45(7):1077-89. http:// dx.doi.org/10.1682/JRRD.2007.11.0197. PMid:19165696.

Peruzzi A, Della Croce U, Cereatti A. Estimation of stride length in level walking using an inertial measurement unit attached to the foot: a validation of the zero velocity assumption during stance. J Biomech. 2011; 44(10):1991-4. http://dx.doi. org/10.1016/j.jbiomech.2011.04.035. PMid:21601860.

Rouhani H, Favre J, Crevoisier X, Aminian K. Ambulatory assessment of $3 \mathrm{D}$ ground reaction force using plantar pressure distribution. Gait Posture. 2010; 32(3):311-6. http://dx.doi. org/10.1016/j.gaitpost.2010.05.014. PMid:20576436.

Savelberg HHCM, Lange ALH. Assessment of the horizontal, fore-aft component of the ground reaction force from insole pressure patterns by using artificial neural networks. Clin Biomech (Bristol, Avon). 1999; 14(8):585-92. http://dx.doi. org/10.1016/S0268-0033(99)00036-4. PMid:10521642.

Zadpoor AA, Nikooyan AA. The relationship between lowerextremity stress fractures and the ground reaction force: a systematic review. Clin Biomech (Bristol, Avon). 2011; 26(1):23-8. http://dx.doi.org/10.1016/j.clinbiomech.2010.08.005. PMid:20846765. 